\title{
Cambios en las comunidades de peces de los arrecifes coralinos del Archipiélago Sabana-Camagüey, Cuba
}

\author{
Rodolfo Claro ${ }^{1}$, Karel Cantelar ${ }^{1}$, Fabián Pina Amargós ${ }^{2}$ \& Juan P. García-Arteaga ${ }^{1}$ \\ 1 Instituto de Oceanología, Ministerio de Ciencia, Tecnología y Medio Ambiente (CITMA). 1ra. No. 18406, Rpto. \\ Flores, Playa, La Habana, Cuba. Fax: (537) 33 9112; rclaro39@yahoo.es \\ 2 Centro de Investigaciones de Ecosistemas Costeros, CITMA, Cayo Coco, Ciego de Ávila, Cuba.
}

Recibido 10-VII-2002. Corregido 21-II-2007. Aceptado 23-II-2007.

\begin{abstract}
Changes in fish communities of coral reefs at Sabana-Camagüey Archipelago, Cuba. A comparison of fish community structure in the Sabana-Camagüey Archipelago (1988-1989 and 2000) using visual census surveys (eight belt transects $2 \times 50 \mathrm{~m}$ in each site) suggests a notable decrease on species richness, and a two thirds reduction in fish density and biomass on coral reefs. This decrease in fish populations may be related to the alarming decrease of scleractinian coral cover, and an enormous proliferation of algae, which currently covers $70-80 \%$ of the hard substrate, impeding the recovery of corals and other benthic organisms. High coral mortalities occurred between the study periods, which correlate with the high temperatures caused by the ENSO events of 1995, 1997 and 1998. These events caused massive bleaching of corals and subsequent algae overgrowth. Evidence of nutrient enrichment from the inner lagoons and overfishing are also present. Collectively, these effects have provoked a marked degradation of reef habitats. These changes appear to have affected the availability of refuges and food for fishes, and may be constraining individual growth potential and population size. Rev. Biol. Trop. 55 (2): 537-547. Epub 2007 June, 29.
\end{abstract}

Key words: fish community, trophic relations, coral reefs, environmental changes, anthropic impacts, Cuba.

El Archipiélago Sabana-Camagüey, también conocido como Jardines del Rey, en los últimos 20 años ha sido objeto de una alta tasa de desarrollo socio-económico, y por tanto sometido a importantes impactos antrópicos. A la actividad pesquera tradicional, se sumó la exploración petrolera y posteriormente el intenso desarrollo del turismo en algunos de los Cayos que bordean la plataforma (Cayos Santa María, Guillermo, Coco, Romano, Cruz y Paredón Grande). Para facilitar el acceso a los mismos, se construyeron varias carreteras a través de la plataforma somera, que unen la isla principal con algunos cayos y estos entre sí, lo cual provocó notables alteraciones del régimen de circulación y cambios en la biota marina (Alcolado et al. 1999). Según Alcolado et al. (2000), ya desde 1994 se detectaron niveles relativamente altos de nutrientes (Fosfatos disueltos) en los arrecifes coralinos de la zona.

Por otra parte, en la década del 90 a escala mundial ocurrieron notables modificaciones del clima que pudieron haber afectado la diversidad biológica de la región. Así por ejemplo, como resultado del calentamiento global se ha incrementado la periodicidad e intensidad de los eventos ENOS, provocando graves afectaciones en los arrecifes coralinos de todo el mundo (Wilkinson 1998).

A fines de la década del 80 , se realizó un inventario de la ictiofauna en los arrecifes y manglares del Archipiélago Sabana-Camagüey (Claro y García-Arteaga 1993, 1994). Se realizaron censos visuales en 31 estaciones en las pendientes arrecifales, entre 15 y $25 \mathrm{~m}$ de profundidad, 25 estaciones en las crestas, 24 en los arrecifes de parche, 63 en los manglares y un 
número menor en otros tipos de hábitat arrecifales. La existencia de tales datos nos permitió evaluar los cambios ocurridos en la estructura de las comunidades de peces mediante su comparación con un inventario similar realizado en el año 2000, y aportar información útil para el manejo de los hábitats costeros del Archipiélago.

Tanto la diversidad como la densidad y biomasa de peces observada en el período 1988-89 (Claro y García-Arteaga 1994) en el Archipiélago Sabana-Camagüey, se consideró relativamente alta, y muy superior a la encontrada por los mismos autores en los arrecifes de las islas Martinica y Guadalupe (Claro et al. 1998), donde la sobrepesca parece haber sido la causa primaria de la baja biomasa encontrada.

Este trabajo tiene como objetivos evaluar el estado de las comunidades de peces en los arrecifes de la región en el año 2000, tomando como referencia los datos obtenidos en similares muestreos realizados en 1988-1989, en un grupo de estaciones distribuidas a lo largo del archipiélago.

\section{MATERIALES Y MÉTODOS}

El estudio se realizó en los arrecifes coralinos del Archipiélago Sabana-Camagûey (en la región norte central de Cuba (entre los 77 y $81^{\circ}$. longitud oeste) en abril del 2000 , mediante censos visuales. En cada sitio se realizaron ocho transeptos lineales de $50 \mathrm{~m}$ de largo por $2 \mathrm{~m}$ de ancho en donde se contabilizaron todas las especies de peces observadas y se anotó la cantidad de individuos y las tallas de cada uno. Las estaciones estuvieron ubicadas en 11 pendientes y nueve crestas arrecifales distribuidas a lo largo del Archipiélago (Fig. 1) Las estaciones fueron seleccionadas entre aquellas muestreadas en el período 1988-1989 con igual método.

Para cada estación se estimaron los valores acumulativos y los promedios \pm la desviación estándar (DE) de densidad y biomasa, así como el de Índice de diversidad de Shannon ( $\left.\mathrm{H}^{\prime}\right)$, el Índice de Riqueza de Margalef $\left(\mathrm{R}_{1}\right)$, el Índice de equitatividad de Pielou (J) (Boesh 1977), el Índice de Jerarquía y el Índice de frecuencia/abundancia (Mason y Brian 1974), estos dos últimos solo para conjuntos de muestras. La riqueza de especies para cada hábitat se evaluó además mediante curvas acumulativas del número de especies por área muestreada, estimadas mediante cinco ó más permutaciones aleatorias de las unidades de muestreo y obteniendo la curva de tendencia promedio para cada grupo de muestras.

El procesamiento de los datos se realizó mediante el programa de computación ESTRUCOM, (elaborado por J. P. GarcíaArteaga y R. Claro, versión 2, 1998) que brinda la siguiente información: lista de especies en orden taxonómico, la densidad y biomasa de cada una y el total para la familia o para la categoría trófica, así como los valores de los mencionados índicadores de la diversidad.

\section{RESULTADOS}

La riqueza de especies en la pendiente arrecifal, expresada por las curvas acumulativas de número de especies por área muestreada (Fig. 2) fue mayor en el período 1988-89. Por otra parte, se observó una notable disminución en la densidad de las especies dominantes, razón por la cual el valor del índice de equitatividad fue mayor en el 2000 (Cuadro 1).

Se aplicó análisis de varianza para comparar los valores de densidad y biomasa entre ambos períodos y se encontró diferencias significativas en ambos casos $(\mathrm{p}<0.01$ y $\mathrm{p}<$ 0.05 respectivamente). Los valores promedios muestran una disminución de aproximadamente dos tercios en ambos parámetros en el año 2000 (Cuadro 1). La Fig. 3 muestra que, en la mayoría de las estaciones, las densidades de peces fueron superiores en el primer inventario, con un incremento sustancial de dicha diferencia hacia la región oriental. La diferencia en biomasa fue aún mayor, manifestándose prácticamente en todas las estaciones (Fig. 4).

La disminución en la densidad de peces se expresó en todos las categorías tróficas (Cuadro 2) con excepción de los omnívoros y 


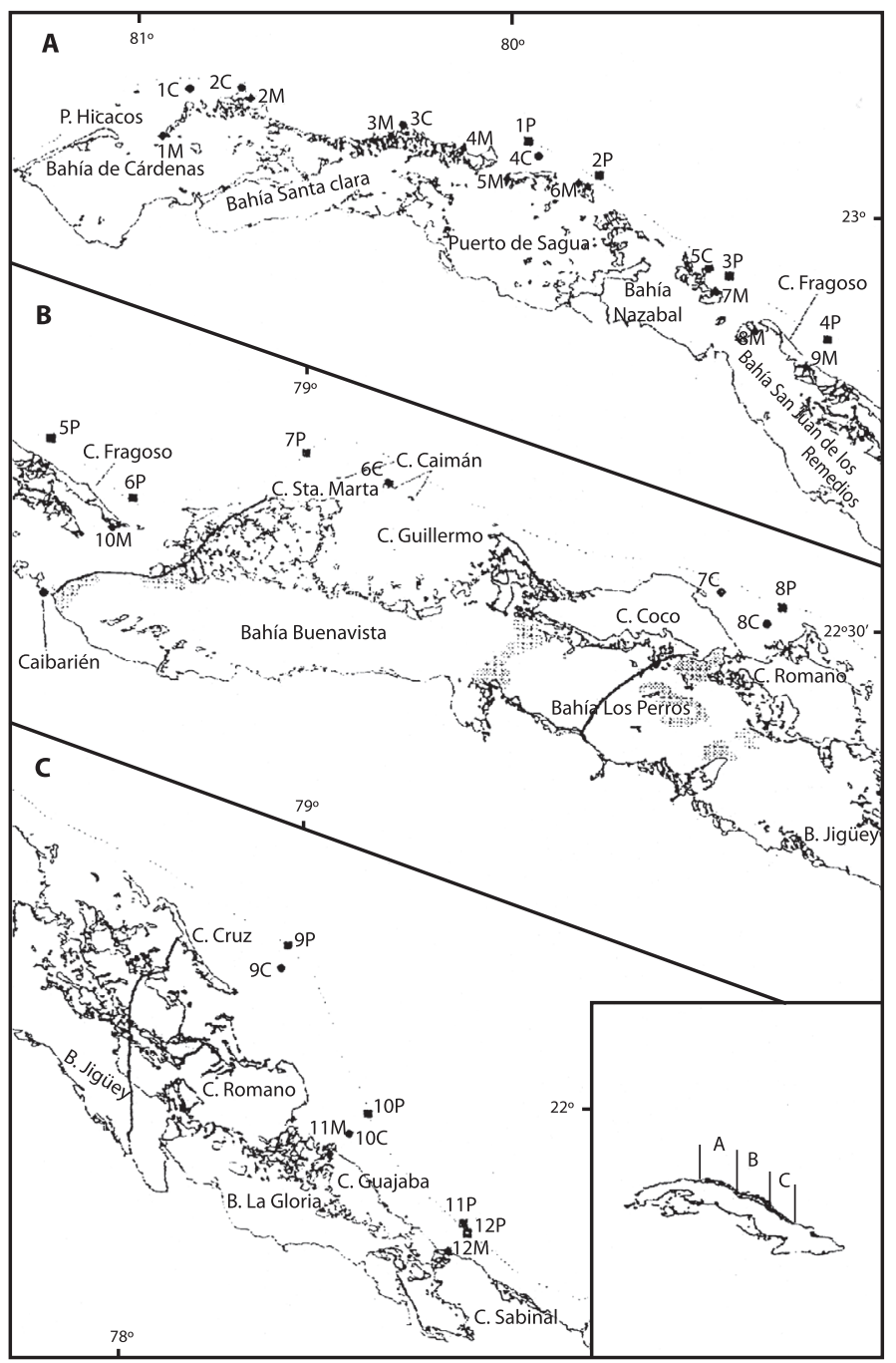

Fig. 1. Localización de los sitios censados en las pendientes arrecifales (P) y en las crestas (C) del Archipiélago SabanaCamagüey, Cuba, en los años 1988-89 y 2000.

Fig. 1. Sites location of censused fore reefs (P) and crest reefs (C) in the Archipelago Sabana-Camagüey, Cuba, in the years 1988-89 and 2000.

fue más notoria en el caso de los planctófagos debido principalmente a la disminución de dos especies dominantes por su número en este hábitat, el cromis azul (Chromis cyanea) y la jenízara (Clepticus parrae), cuyas densidades bajaron en seis veces y sus biomasas en diez. Sin embargo, estos pequeños peces no son determinantes en la biomasa total, la cual refleja mejor los cambios energéticos del ecosistema. La pérdida de biomasa se debió principalmente a la disminución de los peces depredadores bentófagos y piscívoros, entre los que se encuentran las principales especies comerciales. La densidad de tales carnívoros disminuyó sólo en 2.3 veces, pero su biomasa fue 5.3 veces menor (Cuadro 2). La mayor disminución de la biomasa total en relación con la densidad es debida a una reducción de las tallas 
CUADRO 1

Comparación de algunos indicadores de las comunidades de peces en las pendientes arrecifales en 1988-89 y el 2000, Archipiélago Sabana-Camagüey, Cuba

TABLE 1

Comparison of some indicators of fish communities on the slope reefs in the years 1988-89 and 2000, Archipelago Sabana-Camagüey, Cuba

Indicadores

Acumulados

1988-89

2000

10557

303

6175

Área muestreada $\left(\mathrm{m}^{2}\right)$

Densidad (ind. $/ 100 \mathrm{~m}^{2}$ )

24.318

32

118

4.06

8.24

0.59

$\begin{array}{lll}\mathrm{H}^{\prime} & 4.06 & 4.64 \\ \mathrm{R}_{1} & 8.24 & 7.91 \\ \mathrm{~J} & 0.59 & 0.69\end{array}$

Promedios/estación \pm DE

1988-89

2000

$290 \pm 210$

$104 \pm 41$

$27.124 \pm 21.604$

$19 \pm 3$

$7.949 \pm 6.858$

$49 \pm 12$

$18 \pm 3$

$46 \pm 10$

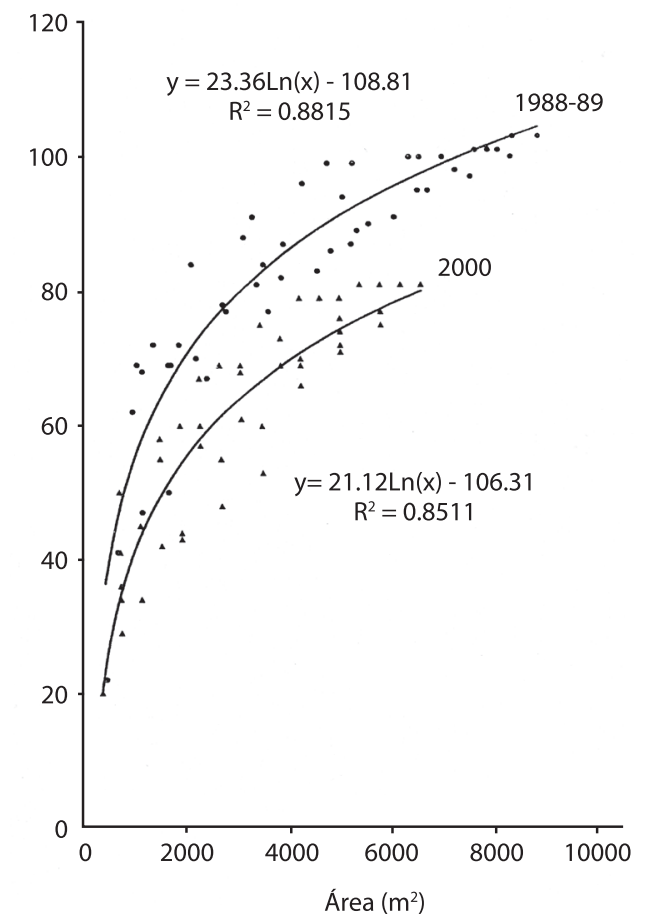

Fig. 2. Comparación de las curvas acumulativas de riqueza de especies en las pendientes arrecifales entre los años 1988-89 y el 2000, Archipiélago Sabana-Camagüey, Cuba.

Fig. 2. Comparison of cumulative curves of species richness on fore reefs in the years 1988-89 and 2000, Archipelago Sabana-Camagüey, Cuba. en la mayoría de las especies, lo cual es un indicio de aumento de la mortalidad total.

Tanto la densidad como la biomasa de herbívoros disminuyeron también, (exceptuando a Microspathodon chrysurus), a pesar del desmesurado crecimiento de las poblaciones de algas. Aunque la densidad de la mayoría de los omnívoros también disminuyó en el año 2000, la densidad de Stegastes partitus, especie dominante en ambos períodos, aumentó notablemente, determinando un incremento de la abundancia de todo el grupo.

En las crestas arrecifales la riqueza de especies mostró similar disminución que en las pendientes (Fig. 5). Los indicadores de diversidad de especies en este hábitat muestran valores inferiores a las pendientes (Cuadros 1 y 3 ).

En las crestas estudiadas del Archipiélago Sabana-Camagüey, Claro y García-Arteaga (1994) reportaron promedios de densidad y biomasa de 269 ind. $/ 100 \mathrm{~m}^{2}$ y $12.628 \mathrm{~kg} / 100 \mathrm{~m}^{2}$ respectivamente, para 24 estaciones muestreadas durante los años 1988-89. La diversidad de especies fue casi tan alta como en el arrecife frontal, aunque con diferentes especies dominantes.

En los muestreos realizados en el año 2000 en las crestas arrecifales, se observó también una disminución de la densidad promedio de 


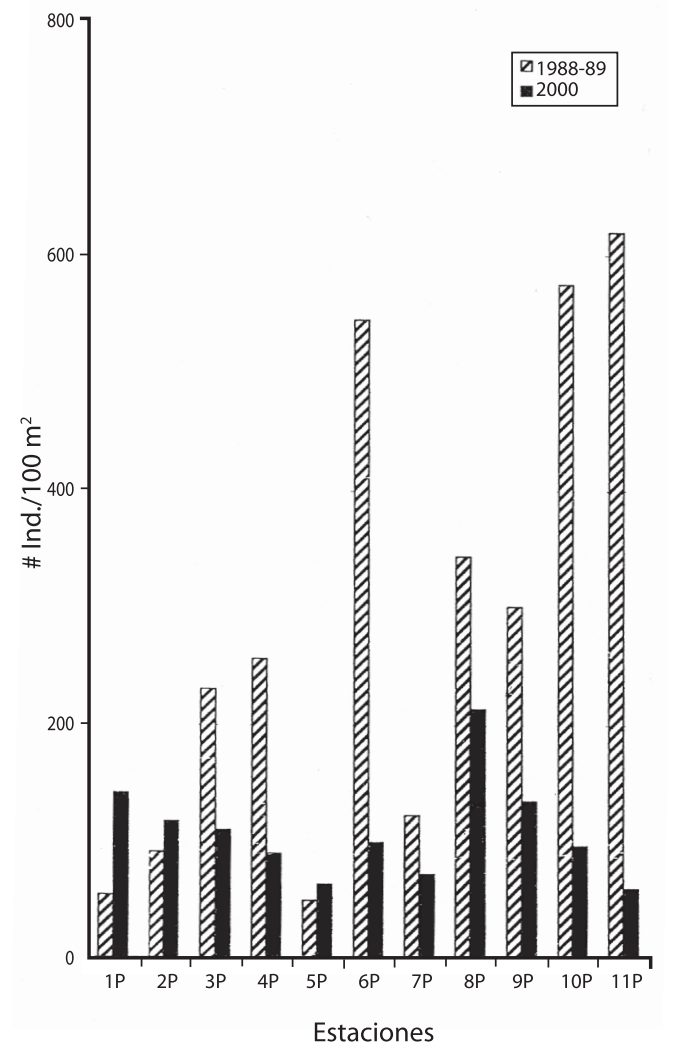

Fig. 3. Comparación de la densidad de peces en las pendientes arrecifales entre los años 1988-89 y el 2000, Archipiélago Sabana-Camagüey, Cuba.

Fig. 3. Comparison of fish density on fore reefs in the years 1988-89 and 2000, Archipelago Sabana-Camagüey, Cuba.

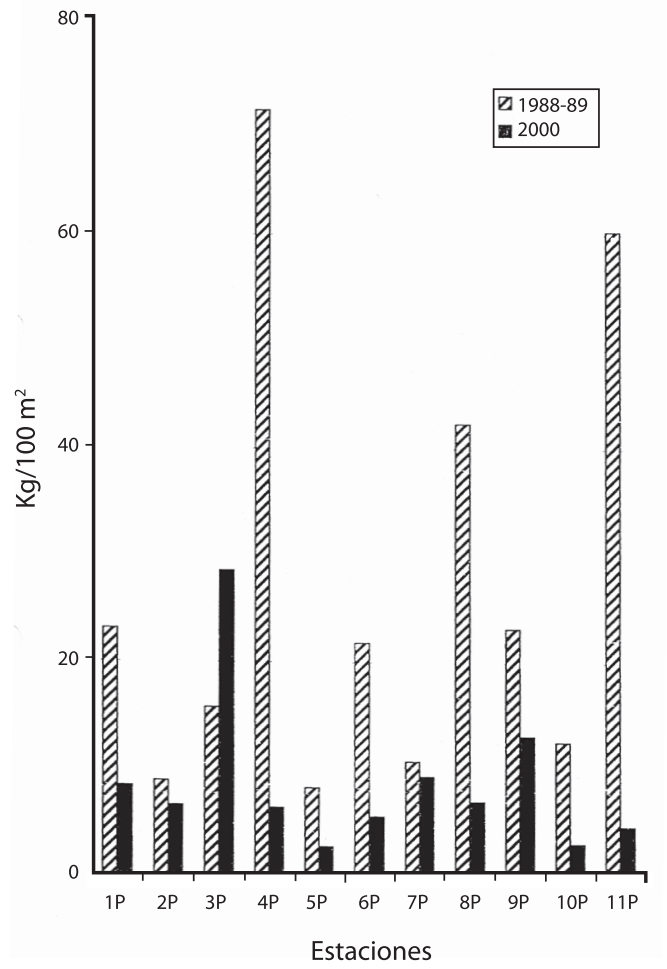

Fig. 4. Comparación de la biomasa de peces en las pendientes arrecifales en los años 1988-89 y el 2000, Archipiélago Sabana-Camagüey, Cuba.

Fig. 4. Comparison of fish biomass on fore reefs in the years 1988-89 and 2000, Archipelago Sabana-Camagüey, Cuba.

\section{CUADRO 2}

Comparación de las densidades y biomasas acumulativas de peces en 11 pendientes arrecifales por categorías tróficas en los años 1988-89 y el 2000, Archipiélago Sabana-Camagüey, Cuba

TABLE 2

Comparison of cumulative fish density and biomass in 11 slope reefs, by trophic categories in the years 1988-89 and 2000, Archipelago Sabana-Camagüey, Cuba

Categorías tróficas

$\begin{array}{lcc} & 1988-89 & 2000 \\ \text { Omnívoros } & 16.46 & 20.97 \\ \text { Herbívoros } & 42.98 & 22.98 \\ \text { Planctófagos } & 169.06 & 26.40 \\ \text { Bentófagos } & 67.10 & 38.50 \\ \text { Piscívoros } & 5.78 & 0.37 \\ \text { Piscívoros-bentófagos } & 12.23 & 4.72\end{array}$

$$
\text { Biomasa }\left(\mathrm{kg} / 100 \mathrm{~m}^{2}\right)
$$

$\begin{array}{cc}1988-89 & 2000 \\ 0.08 & 0.06 \\ 3.43 & 2.24 \\ 5.06 & 0.49 \\ 5.46 & 3.51 \\ 7.77 & 0.79 \\ 8.19 & 1.52\end{array}$



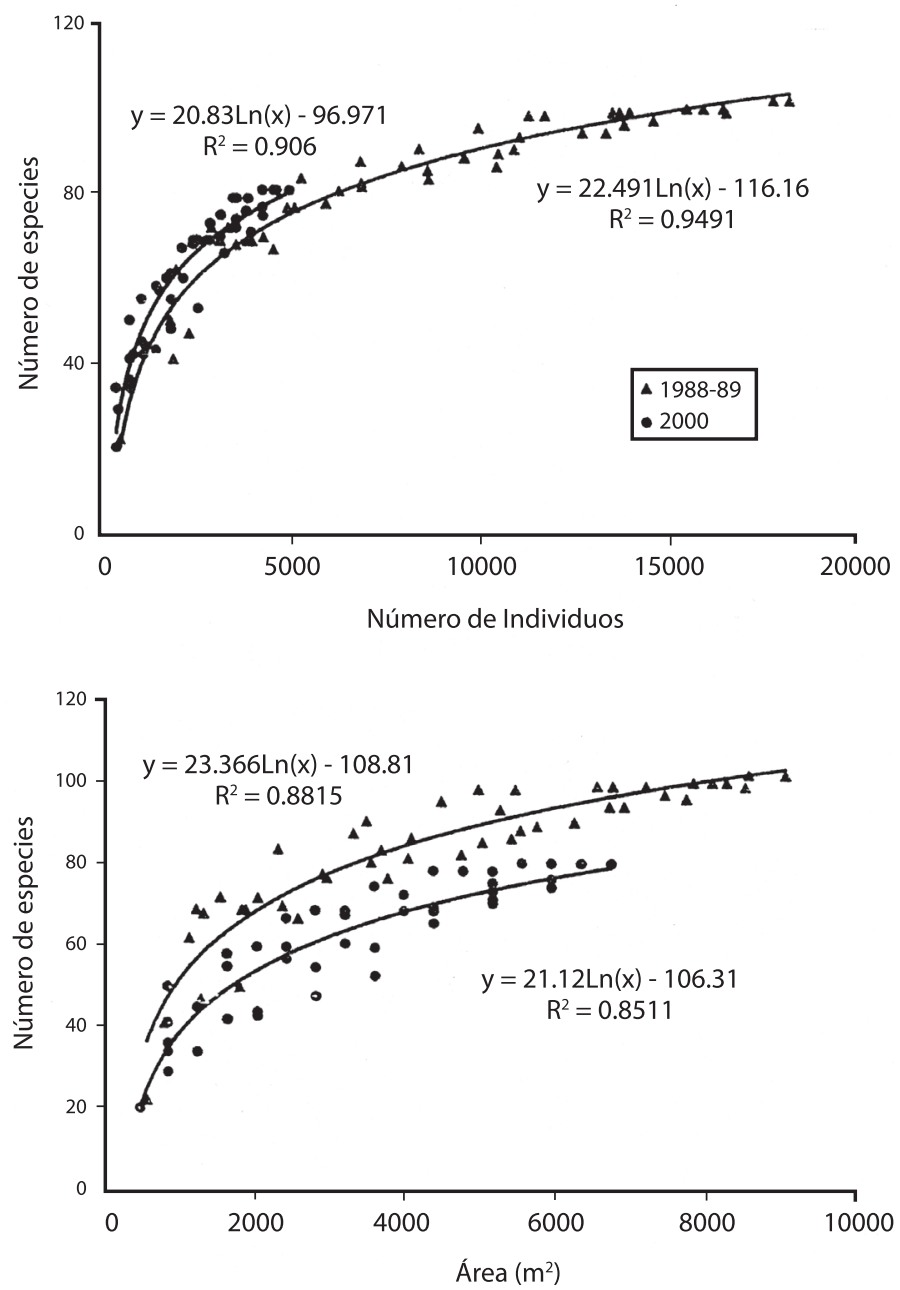

Fig. 5 Comparación de las curvas acumulativas de riqueza de especies en las crestas arrecifales en los años 1988-89 y el 2000, Archipiélago Sabana-Camagüey, Cuba.

Fig. 5. Comparison of cumulative curves of species richness on crest reefs in the years 1988-89 and 2000, Archipelago Sabana-Camagüey, Cuba.

$66 \%$ y $54 \%$ de su biomasa promedio (Cuadro 3), siendo significativa la diferencia en ambos casos según los análisis de varianza realizados para ambas variables $(\mathrm{p}<0.05$ en ambos casos). Similar disminución muestran los valores acumulativos de esos parámetros. Al igual que en las pendientes, tal reducción ocurrió en casi todas las estaciones (Fig. 6 y 7) con mayores disminuciones de la biomasa en la región central y oriental.
Tanto la densidad como la biomasa de peces en las crestas, disminuyeron en el año 2000, en todas las categorías tróficas (Cuadro 4). La diferencia fue particularmente notable en los herbívoros, debido a la reducción en la abundancia de los barberos (Acanthurus spp.) y a la casi desaparición de los herbívoros de gran talla, como el loro guacamayo (Scarus guacamaia), y el loro colirrojo, Sparisoma chrysoptera. Entre los bentófagos también se 
CUADRO 3

Comparación de algunos indicadores de las comunidades de peces en las crestas arrecifales en los años 1988-89 y el 2000, Archipiélago Sabana-Camagüey, Cuba

TABLE 3

Comparison of some indicators of fish communities on crest reefs in the years 1988-89 and 2000, Archipelago Sabana-Camagüey, Cuba

Parámetros

Area Muestreada $\left(\mathrm{m}^{2}\right)$
Densidad (ind. $\left./ 100 \mathrm{~m}^{2}\right)$
Biomasa $\left(\mathrm{kg} / 100 \mathrm{~m}^{2}\right)$
No. de familias
No. de especies
H'
R1
J

Valores acumulativos

$\begin{array}{cc}1988-89 & 2000 \\ 9180 & 6800 \\ 200 & 70 \\ 10.37 & 4.742 \\ 33 & 25 \\ 103 & 83 \\ 4.84 & 4.30 \\ 7.20 & 6.46 \\ 0.72 & 0.68\end{array}$

Valores promedios $\pm \mathrm{DE}$ 1988-89 2000

$9180 \quad 6800$

$211 \pm 87 \quad 71 \pm 22$

$\begin{array}{ll}10.550 \pm 5.227 & 4.872 \pm 3.791\end{array}$

$18 \pm 5 \quad 14 \pm 4$

$55 \pm 16 \quad 40 \pm 16$

\section{CUADRO 4}

Comparación de las densidades y biomasas de peces en las pendientes arrecifales por categorías tróficas en los años 1988-89 y el 2000, Archipiélago Sabana-Camagüey, Cuba

TABLE 4

Comparison of fish density and biomass on the crest reefs, by trophic categories in the years 1988-89 and 2000, Archipelago Sabana-Camagüey, Cuba

Categorías tróficas

Densidad (Ind./100 $\mathrm{m}^{2}$ ) 1988-89

Omnívoros

Herbívoros

Planctofagos

Bentofagos

Piscívoros

Piscívoros-Bentofagos

\subsection{7}

113.72

4.51

6.62
2000

9.06

17.88

0.35

41.08

0.61

1.4
Biomasa $\left(\mathrm{kg} / 100 \mathrm{~m}^{2}\right)$

$\begin{array}{cc}1988-89 & 2000 \\ 0.070 & 0.049 \\ 4.371 & 1.067 \\ 0.130 & 0.002 \\ 3.419 & 1.655 \\ 1.233 & 0.785 \\ 1.144 & 0.236\end{array}$

observó una disminución grande, ocasionada en gran medida por la pérdida en la abundancia de roncos (Haemulidae) en el año 2000.

\section{DISCUSIÓN}

En los muestreos realizados en el año 2000 se observó una gran cantidad de corales escleractínios y gorgonáceos muertos, tanto en las pendientes como en las crestas arrecifales. La mayoría de los corales muertos estaban cubiertos por algas y organismos incrustantes, lo cual evidencia que tal mortandad no es reciente. Los corales vivos eran escasos (solo $10-20 \%$ del fondo cubierto por estos), aunque en buen estado de salud (pocos blanqueados o con enfermedades). Sin embargo, en casi 


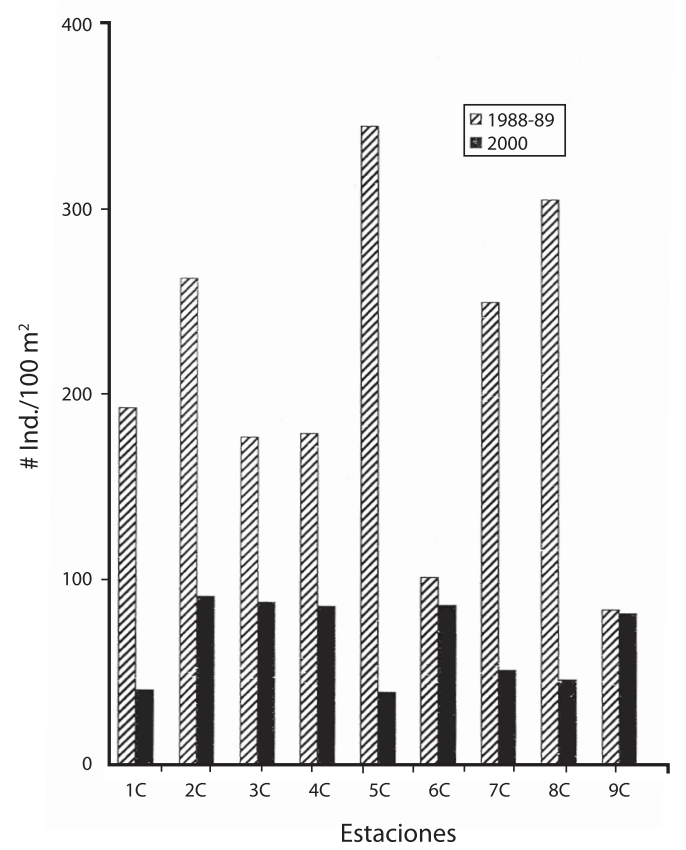

Fig. 6. Comparación de la densidad de peces en las crestas arrecifales en 1988-89 y el 2000, Archipiélago SabanaCamagüey, Cuba.

Fig. 6. Comparison of fish density on crest reefs in the years 1988-89 and 2000, Archipelago Sabana-Camagüey, Cuba.

todas las estaciones el $60-80 \%$ del substrato duro estaba cubierto por una densa alfombra de algas, principalmente Dictyosphaeria cavernosa, Lobophora variegata, Cladophora spp., Halimeda spp., Penicillus spp. y Stypopodium zonale. En los años 1988-89, en ninguna estación se observó semejante cubrimiento algal. Similar situación fue encontrada por dos de los observadores durante muestreos realizados en 1991 en las islas Martinica y Guadalupe (Claro et al. 1998) donde ya en ese período se había producido una gran disminución de la biomasa de peces y un aumento inusitado de las algas, probablemente como consecuencia de la desaparición del erizo negro (Diadema antillarum) y de la sobrepesca de los peces herbívoros.

Al parecer, la disminución de la cobertura de corales y el aumento del cubrimiento de algas en los arrecifes coralinos, parece ser consecuencia de la elevación de la temperatura

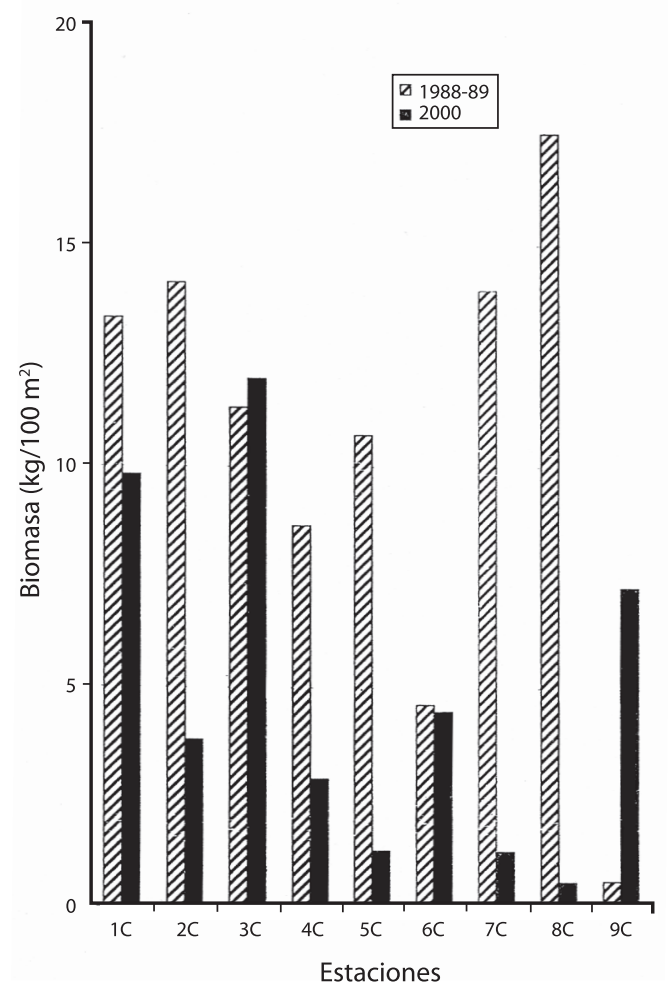

Fig. 7. Comparación de la biomasa de peces en las crestas arrecifales en 1988-89 y el 2000, Archipiélago SabanaCamagüey, Cuba.

Fig. 7. Comparison of fish biomass on crest reefs in the years 1988-89 and 2000, Archipelago Sabana-Camagüey, Cuba.

como resultado de cambios climáticos. Después del evento "El Niño", que afectó el Archipiélago Cubano en 1995, Carrodeguas et al. (1996) reportaron gran mortalidad de corales por blanqueamiento. Otros dos eventos más intensos ocurrieron en 1997 (El Niño) y 1998 (La Niña), cuando se registraron marcadas anomalías térmicas positivas al norte del Archipiélago según se observa en una imagen satelitaria que promedia el período Enero-Junio de 1998 (Wilkinson 1998), y en los gráficos de temperatura superficial del mar al norte y sur de Cuba durante el período Junio de 1981 a Junio del 2000 (Cerdeira et al., 2005).

Por otra parte, ya desde 1994 se detectaron concentraciones de nutrientes relativamente 
altos en la región, aparentemente provocadas por la afluencia desde las bahías eutroficadas, las cuales reciben fuertes descargas de materia orgánica desde las industrias azucareras y, residuales de las plantas de levadura torula, entre otras fuentes de eutroficación (Alcolado et al. 1999, Alcolado et al, 2000). Por otro lado, algunas imágenes satelitarias muestran que las aguas del Canal Viejo de Bahamas están ligeramente más nutrificadas (mayor concentración de pigmentos) que otras aguas oceánicas adyacentes a la plataforma Cubana. Ello puede ser resultado de una menor dinámica de esas aguas (R. Pérez comunicación personal). La mayor capacidad de renovación de las aguas en el Canal de Bahamas podría incrementar la influencia de esas aguas enriquecidas sobre las plataformas de Cuba y Bahamas (Alcolado et al. 2000) lo cual podría facilitar la invasión de las algas sobre los corales blanqueados.

La densidad de peces herbívoros disminuyó grandemente a pesar de la gran proliferación de algas. Esta última no implica necesariamente una mejoría de su base alimentaria. Muchas, de tales algas, son disuasivas para los peces o no son consumidas por estos por otros motivos. Generalmente con el tamaño y densidad de la alfombra algal disminuye la posibilidad de ser consumidas por los herbívoros (Steneck y Watling 1982, Littler et al. 1983, Hay 1991). Los loros (Scaridae), por ejemplo, se alimentan de las pequeñas algas epífitas que crecen sobre los corales o las rocas, pero raramente consumen las algas crustosas y otras de mayor tamaño, a veces altamente calcificadas. Los barberos (Acanthuridae) pueden consumir las algas más grandes, pero también evaden aquellas que acumulan carbonatos. Varias de las especies de algas abundantes en las estaciones muestreadas se encuentran entre las listadas por Hay (1991) como altamente disuasivas para los peces herbívoros.

La disminución en la abundancia de corales escleractínios implica una severa reducción de la disponibilidad de refugios tanto para los peces como para los organismos bentónicos que sirven de alimento a estos. Aún los planctívoros diurnos que se alimentan en la columna de agua, como el Chromis azul (Chromis cyanea), dependen de la existencia de refugios en el arrecife para protegerse de sus depredadores u otros peligros y para descansar (Hobson 1991).

La existencia de refugios, que brindan los corales y otros organismos sésiles asociados a estos, constituye un elemento crítico para los procesos ecológicos que se encuentran en el arrecife, y su ausencia o disminución podrían determinar una reducción de la productividad biológica de todo el ecosistema. Tal reducción se reflejaría de inmediato en la densidad y biomasa de los peces, que son los principales usuarios de tales recursos.

Otros elementos a considerar, son la escasez de erizos negros (Diadema antillarum), (provocada por una pandemia en todo el Caribe a partir de 1983) y de los peces herbívoros, en el caso de estos últimos como resultado de la sobrepesca reportada para la zona (Claro et al. 2000).

Los datos obtenidos demuestran una notable disminución de las tallas de los peces en el año 2000 en relación con período 1988-89, que se refleja principalmente en los depredadores piscívoros (Cuadro 2). Ello parece ser consecuencia del incremento del esfuerzo pesquero, en particular la pesca no comercial, principalmente la que se realiza con arpón neumático, la cual aumentó extraordinariamente durante la década del 90 (Claro et al. 2000). La adopción de nuevas regulaciones pesqueras y el establecimiento de un sistema de vigilancia, han mejorado esa situación, pero sus consecuencias aún se mantienen.

La situación actual de deterioro de los hábitat marinos del Archipiélago SabanaCamagüey, requiere del establecimiento inmediato de un manejo sostenible de la pesca y la implementación de una red de áreas protegidas marinas. Una primera propuesta se elaboró por el Proyecto CUBA-GEF/UNDP "Por un manejo sostenible del ecosistema SabanaCamagüey" (Alcolado et al 1999), y Claro (2001) aportó otros elementos para su diseño. Estas y otras informaciones sirvieron de base para la elaboración, por el Centro Nacional de Áreas Protegidas, de una propuesta al gobierno para el establecimiento de una red de áreas 
protegidas marinas en la región. Un análisis más detallado sobre los impactos de la degradación de los hábitats y la sobrepesca se presenta en Claro et al. (2004) con recomendaciones concretas para cambiar las estrategias de pesca y eliminar los artes destructivos (chinchorros, redes de sitio) y la pesca sobre las agregaciones de desove. Algunas de tales recomendaciones se encuentran ya en proceso de aplicación por el Ministerio de Industria Pesquera.

\section{AGRADECIMIENTOS}

Los autores desean agradecen a Pedro M. Alcolado la revisión crítica de este trabajo, así como la ayuda técnica de Jorge L. Hernández López y los buzos, Armando Pérez Prieto y Benito García Salun. Agradecemos la colaboración de la tripulación del barco de investigaciones Poseidón. Este proyecto fue financiado por el Programa Nacional de Ciencia y Técnica: Cambios Globales y Evolución del Medio Ambiente Cubano, Ministerio de Ciencia, Tecnología y Medio Ambiente, Cuba.

\section{RESUMEN}

Al comparar la estructura de las comunidades de peces en los arrecifes del Archipiélago Sabana-Camagüey entre 1988-89 y el año 2000, mediante iguales censos visuales (ocho transeptos lineales de $2 \times 50 \mathrm{~m}$ en cada estación), se observó una notable disminución de la riqueza de especies y una reducción en dos tercios de la densidad y biomasa de peces. Tal disminución de las poblaciones de peces parece estar relacionada con la alarmante disminución de la cobertura de corales escleractínios y una gran proliferación de algas, las cuales actualmente cubren 70$80 \%$ del sustrato duro, impidiendo la recuperación de los corales y otros organismos bénticos. Grandes mortalidades de corales ocurrieron entre los dos periodos de estudio, lo cual parece ser consecuencia de las altas temperaturas provocadas por los eventos ENOS de 1995, 1997 y 1998. Estos fenómenos climáticos provocaron el blanqueamiento masivo de los corales y como consecuencia se produjo una gran proliferación de las algas. Asimismo en el período entre ambos muestreos ocurrió el enriquecimiento de nutrientes desde las lagunas interiores y la sobreexplotación de los recursos pesqueros. El conjunto de estos factores provocaron una marcada degradación del hábitat en los arrecifes.
Tales cambios parecen haber afectado la disponibilidad de refugios y la alimentación para los peces, limitando el crecimiento individual y el tamaño de las poblaciones.

Palabras clave: comunidad íctica, relaciones tróficas, arrecifes coralinos, cambios ambientales, Cuba.

\section{REFERENCIAS}

Alcolado, P. M., E. E. García y N. Espinosa (eds.). 1999. Protecting biodiversity and establishing sustainable development in the Sabana-Camagüey ecosystem. Global Environmental Facility (GEF/United Nations Development program (UNDP) Project SabanaCamagüey, CUB/92/G31, Cuba. 145 p.

Alcolado, P. M., R. Claro, B. Martínez-Daranas, G. Menéndez-Macías, P. García-Parrado, K. CantelarRamos, J. Espinosa Sáez, R. del Valle, J. C. Martínez Iglesias y T. Neff. 2000. Estado general de los arrecifes coralinos de Cuba y propuestas de manejo ambiental. Informe Final de Proyecto, Instituto de Oceanología, CITMA, La Habana, Cuba. 66 p.

Boesch, D.F. 1977. Application of numerical classifications in ecological investigations of water pollution. Ecological Res. Ser., EPA-GOO/3-77-033, 115 p.

Carrodeguas, C., G. Arencibia, N. Capetillo y M. García. 1996. Decoloración de corales en el Archipiélago Cubano. Centro de Investigaciones Pesqueras, MIP, La Habana, Cuba. 11 p.

Cerdeira-Estrada, S., F.E. Müller-Karger, A. Gallegos. 2005. Variability of the Sea Surface Temperature around Cuba. Gulf of Mexico Science (GOMS), 23:161-171.

Claro, R., y J. P. García-Arteaga. 1993. Estructura de las comunidades de peces asociados a los manglares del grupo insular Sabana-Camagüey, Cuba. Avicennia 0: 60-82.

Claro, R., y J. P. García-Arteaga. 1994. Estructura de las comunidades de peces en los arrecifes del grupo insular Sabana-Camaguey, Cuba. Avicennia 2: 83-107.

Claro, R., J. P. García-Arteaga, y Bouchón-Navarro, M. Louis y C. Bouchon. 1998. La estructura de las comunidades de peces en los arrecifes de las Antillas Menores y Cuba. Avicennia 8/9: 69-86.

Claro, R., K. Cantelar-Ramos, F. Pina-Amargós, y J. P. García-Arteaga. 2000. Biodiversidad y manejo de la ictiofauna del Archipiélago Sabana-Camagüey. Informe de proyecto, Instituto de Oceanología, La Habana, Cuba. 89 p. 
Claro, R. 2001. Propuesta de reservas de pesca y medidas de manejo basada en las congregaciones de desove de pargos y meros en Cuba. Instituto de Oceanología, CITMA, La Habana, Cuba. 16 p.

Claro, R. and L. R. Parenti. 2001. The Marine Ichthyofauna of Cuba. p. 21-32 In R. Claro, K. C. Lindeman y L. R. Parenty (eds.), Ecology of The Marine Fishes of Cuba, Smithsonian Institution, Washington, D.C., USA.

- Claro, R. J. P. García-Arteaga, B. Gobert, K. Cantelar Ramos, S. V. Valle Gómez y F. Pina Amargós. 2004. Situación actual de los recursos pesqueros del Archipiélago Sabana-Camagüey, Cuba. Bol. Invest. Mar. Cost. 33:41-57

Hay, M. E. 1991. Fish-seaweed interaction on coral reefs: effects of herbivorous fishes and adaptations of their prey. p. 86-119 In P. F. Sale (ed.) The Ecology of Fishes on Coral Reefs. Academic Press Inc. San Diego, California, EEUU.
Hobson, E. S. 1991. Trophic relationships of fishes specialized to feed zooplankters above coral reefs. p. 69-95 In P. F. Sale (ed.) The Ecology of Fishes on Coral Reefs. Academic Press Inc. San Diego, California, EEUU.

Littler, M. M., P. R. Taylor \& D.S. Littler. 1983. Algal resistance to herbivory on a Caribbean barrier reef. Coral Reefs 2: 111-118.

Mason, C. F. \& R. J. Bryant 1974. The structure and diversity of the animal communities in a broadland reedswamp. J. Zool. 172: 289-302.

Steneck, R. S. \& L. Watling. 1982. Feeding capabilities and limitations of herbivorous mollusks: a functional group approach. Mar. Biol. 68: 299-319.

Wilkinson, C. 1998. Status of coral reefs of the world. Global Coral Reef Monitoring Network. Australian Institute of Marine Science. Sidney, Australia. $184 \mathrm{p}$. 
